ORIGINAL ARTICLE

\section{Darolutamide in Nonmetastatic, Castration-Resistant Prostate Cancer}

\author{
Karim Fizazi, M.D., Neal Shore, M.D., Teuvo L. Tammela, M.D., Ph.D., \\ Albertas Ulys, M.D., Egils Vjaters, M.D., Sergey Polyakov, M.D., \\ Mindaugas Jievaltas, M.D., Murilo Luz, M.D., Boris Alekseev, M.D., Iris Kuss, M.D., \\ Christian Kappeler, Ph.D., Amir Snapir, M.D., Ph.D., Toni Sarapohja, M.Sc., \\ and Matthew R. Smith, M.D., Ph.D., for the ARAMIS Investigators*
}

ABSTRACT

BACKGROUND

Darolutamide is a structurally unique androgen-receptor antagonist that is under development for the treatment of prostate cancer. We evaluated the efficacy of darolutamide for delaying metastasis and death in men with nonmetastatic, castrationresistant prostate cancer.

\section{METHODS}

We conducted a randomized, double-blind, placebo-controlled, phase 3 trial involving men with nonmetastatic, castration-resistant prostate cancer and a prostate-specific antigen doubling time of 10 months or less. Patients were randomly assigned in a 2:1 ratio to receive darolutamide (600 $\mathrm{mg}$ [two $300-\mathrm{mg}$ tablets] twice daily) or placebo while continuing androgen-deprivation therapy. The primary end point was metastasis-free survival, with the presence of metastasis determined by independent central review of radiographic imaging every 16 weeks.

RESULTS

In total, 1509 patients underwent randomization (955 to the darolutamide group and 554 to the placebo group). In the planned primary analysis, which was performed after 437 primary end-point events had occurred, the median metastasis-free survival was 40.4 months with darolutamide, as compared with 18.4 months with placebo (hazard ratio for metastasis or death in the darolutamide group, $0.41 ; 95 \%$ confidence interval, 0.34 to $0.50 ; \mathrm{P}<0.001$ ). Darolutamide was also associated with benefits with regard to all secondary end points, including overall survival, time to pain progression, time to cytotoxic chemotherapy, and time to a symptomatic skeletal event. The incidence of adverse events that occurred or worsened during the treatment period and had a frequency of 5\% or more or were of grade 3 or higher was similar in the two groups; all such events except fatigue occurred in less than $10 \%$ of patients in either group. The percentage of patients who discontinued the assigned regimen because of adverse events was $8.9 \%$ in the darolutamide group and $8.7 \%$ in the placebo group. Darolutamide was not associated with a higher incidence of seizures, falls, fractures, cognitive disorder, or hypertension than placebo.

\section{CONCLUSIONS}

Among men with nonmetastatic, castration-resistant prostate cancer, metastasis-free survival was significantly longer with darolutamide than with placebo. The incidence of adverse events was similar for darolutamide and placebo. (Funded by Bayer HealthCare and Orion Pharma; ARAMIS ClinicalTrials.gov number, NCT02200614.)
From Institut Gustave Roussy, Université Paris-Sud, Villejuif, France (K.F.); Carolina Urologic Research Center, Myrtle Beach, SC (N.S.); Tampere University Hospital and University of Tampere, Tampere (T.L.T.), and Orion Pharma, Orion Corporation, Espoo (A.S., T.S.) - all in Finland; National Cancer Institute, Vilnius (A.U.), and Medical Academy, Lithuanian University of Health Sciences, Kaunas (M.J.) - both in Lithuania; Stradins Clinical University Hospital, Riga, Latvia (E.V.); N.N. Alexandrov National Cancer Center of Belarus, Minsk, Belarus (S.P.); Hospital Erasto Gaertner, Curitiba, Brazil (M.L.); National Medical Research Radiological Center, Ministry of Health of the Russian Federation, Moscow (B.A.); Bayer, Berlin (I.K., C.K.); and Massachusetts General Hospital Cancer Center, Boston (M.R.S.). Address reprint requests to Dr. Fizazi at the Department of Cancer Medicine, Institut Gustave Roussy, 114 Rue Edouard Vaillant, 94800 Villejuif, France, or at karim.fizazi@gustaveroussy.fr.

*The complete list of ARAMIS investigators is provided in the Supplementary Appendix, available with the full text of this article at NEJM.org.

This article was published on February 14, 2019, at NEJM.org.

N Engl J Med 2019;380:1235-46. DOI: 10.1056/NEJMoal815671

Copyright (C) 2019 Massachusetts Medical Society. 
NDROGEN-DEPRIVATION THERAPY IS part of the standard of care for patients whose prostate cancer recurs after primary treatment. ${ }^{1-4}$ However, despite receiving androgen-deprivation therapy, most of these patients will have disease progression, initially manifesting as rising levels of prostate-specific antigen (PSA). ${ }^{5}$ Some of these patients have an absence of metastases on conventional imaging and are classified as having nonmetastatic, castration-resistant prostate cancer. ${ }^{6}$ Delaying the development of metastases in these patients is a key therapeutic goal, since metastasis in bone, soft tissue, or viscera is associated with both morbidity and prostate cancer-specific mortality. ${ }^{7-9}$

The androgen-receptor inhibitors apalutamide and enzalutamide have recently been approved for the treatment of nonmetastatic, castration-resistant prostate cancer on the basis of phase 3 trials showing significantly longer metastasis-free survival with these agents than with placebo. ${ }^{10,11}$ Data on overall survival or pain associated with these agents are still immature. However, patients with nonmetastatic, castration-resistant prostate cancer, who may have adverse effects from their ongoing androgendeprivation therapy, may have additional associated adverse events and toxic effects from these agents. Thus, there is a need for therapies with improved safety and toxicity profiles.

Darolutamide is an androgen-receptor antagonist with a distinct structure that offers a potential for fewer and less severe toxic effects than apalutamide and enzalutamide because of its low penetration of the blood-brain barrier ${ }^{12-14}$ and low binding affinity for $\gamma$-aminobutyric acid type A receptors, as shown in preclinical studies. ${ }^{15}$ After observing the significant antitumor activity and good side-effect profile shown in phase 1 and 2 studies involving men with metastatic, castration-resistant prostate cancer, ${ }^{16-19}$ we conducted the multinational, randomized, double-blind, placebo-controlled, phase 3 Androgen Receptor Antagonizing Agent for Metastasis-free Survival (ARAMIS) trial to evaluate the efficacy and safety of darolutamide in men with nonmetastatic, castration-resistant prostate cancer. The primary end point was metastasis-free survival, an established end point in trials involving castration-resistant prostate cancer.
METHODS

\section{TRIAL DESIGN AND CONDUCT}

The trial was sponsored by Orion Pharma and Bayer HealthCare; both sponsors developed the trial design with the first and last authors. The trial was conducted in 36 countries worldwide in 409 centers. The institutional review board at each participating institution approved the trial, which was conducted in compliance with the principles of the Declaration of Helsinki and in accordance with the International Conference on Harmonisation guidelines for Good Clinical Practice. All patients provided written informed consent. An independent data and safety monitoring board reviewed unblinded safety data throughout the trial. The data were collected by the investigators, analyzed by statisticians who were employed by the sponsors, and interpreted by the authors, including employees of the sponsors. Bayer HealthCare provided funding for medical writing and editing assistance. The authors reviewed and approved the manuscript that was submitted for publication. The authors assume responsibility for the completeness and accuracy of the data and for the fidelity of the trial and this report to the protocol and statistical analysis plan, available with the full text of this article at NEJM.org.

\section{PATIENTS}

Patients were eligible for participation if they were 18 years of age or older and had histologically or cytologically confirmed adenocarcinoma of the prostate. Patients were required to have castration-resistant prostate cancer, a baseline PSA level of at least 2 ng per milliliter, a PSA doubling time of 10 months or less, and an Eastern Cooperative Oncology Group (ECOG) performance status of 0 or 1 (scores range from 0 to 5 , with higher numbers reflecting greater disability). At screening, all patients underwent a radionuclide bone scan of the whole body and computed tomography (CT) or magnetic resonance imaging (MRI) of the pelvis, abdomen, and chest; patients with detectable metastases or a history of metastatic disease were excluded, although the presence of pelvic lymph nodes less than $2 \mathrm{~cm}$ in diameter in the short axis below the aortic bifurcation was allowed. Previous seizure or conditions predisposing to seizure were not exclusion- 
ary. Full details of the criteria for patient selection are provided in the Supplementary Appendix, available at NEJM.org.

\section{TRIAL DESIGN AND REGIMENS}

At trial initiation, patients were randomly assigned in a 2:1 ratio in a double-blind manner to receive either darolutamide (600 mg given as two 300-mg tablets) twice daily with food (a daily dose of $1200 \mathrm{mg}$ ) or matched placebo. Randomization was stratified according to PSA doubling time ( $\leq 6$ months or $>6$ months) and the use of osteoclast-targeted therapy at randomization (yes or no). Patients continued taking the randomly assigned regimen until protocol-defined progression, discontinuation of the regimen because of adverse events, or withdrawal of consent. Patients continued to receive androgen-deprivation therapy (luteinizing hormone-releasing hormone agonist or antagonist) throughout the trial. Patients who initiated a prohibited therapy (listed in the protocol) before confirmation of metastasis had to discontinue the trial regimen and were followed for survival status.

\section{ASSESSMENTS}

Information on the patients' demographic characteristics, relevant medical history, and pertinent clinical conditions was obtained at screening. Data on vital signs and laboratory safety assessments were obtained at the trial research center at screening and at every scheduled visit (at days 1,15 , and 29; at 16 weeks; and at 16-week intervals thereafter). Serum PSA level and pain (evaluated with the use of the Brief Pain Inventory Short-Form [BPI-SF] questionnaire, a 10-point scale on which higher numbers reflect greater pain; minimum clinically important difference, 2 points) were assessed at screening, day 1 , week 16 , and every subsequent visit until the end of the trial or death. Health-related quality-of-life instruments were assessed at screening, day 1 , week 16 , and the end of the treatment period with the Functional Assessment of Cancer Therapy-Prostate (FACT-P; the total score is the sum of the scores of 39 items of the questionnaire and ranges from 1 to 156, with higher scores indicating better quality of life; minimum clinically important difference, 10 points), the prostate cancer-specific subscale of the FACT-P (FACT-P PCS; minimum clinically important difference, 3 points), ${ }^{20}$ and the generic EuroQol Group 5-dimension 3-level (EQ-5D-3L; five dimensions, each with three levels of response, are summarized as an index score ranging from -0.59 to 1 , with higher scores indicating better health states; minimum clinically important difference, 0.06 points); the FACT-P PCS was also given every 16 weeks until the end of the trial or death. The European Organisation for Research and Treatment of Cancer quality of life questionnaire urinary symptoms subscale (EORTC-QLQ-PR25, a 25-item questionnaire on which higher scores indicate a greater effect of symptoms on quality of life; minimum clinically important difference, 8 points) ${ }^{21}$ was given at screening, day 1 , week 16 , and every 16 weeks until the end of the treatment period. Disease assessments - including evaluation of ECOG performance status, bone scans, and CT and MRI of the chest, abdomen, and pelvis - were performed at screening, week 16, and every subsequent 16-week visit. All scans were evaluated both locally and by blinded independent central review.

Data on adverse events that occurred or worsened during the treatment period, including the type, severity (according to the National Cancer Institute Common Terminology Criteria for Adverse Events, version 4.03), ${ }^{22}$ and seriousness of the events and whether they were assessed by the investigator as being related to the trial regimen, were recorded at each visit. Safety was evaluated in all patients who underwent randomization and received at least one dose of darolutamide or placebo.

\section{END POINTS}

The primary end point was metastasis-free survival, defined as the time from randomization to confirmed evidence of distant metastasis on imaging or death from any cause, whichever occurred first. The blinded central imaging review for efficacy was performed by a pool of radiologists separate from those who performed the blinded central imaging review for eligibility. During the central efficacy imaging review, which included the baseline scans, some patients were retrospectively classified as having metastases at baseline. These patients were included in the primary analysis of metastasis-free survival. Sensitivity analyses are summarized in the Supplementary Appendix.

The secondary end points were overall survival, time to pain progression (defined as either 
an increase of $\geq 2$ points from baseline in the score assessed with the BPI-SF questionnaire or initiation of opioid treatment for cancer pain, whichever occurred first), time to first symptomatic skeletal event (defined as external-beam radiation therapy to relieve skeletal symptoms, new symptomatic pathologic bone fracture, occurrence of spinal cord compression, or tumor-related orthopedic surgical intervention), and time to first cytotoxic chemotherapy.

Exploratory end points included progressionfree survival (defined as the time from randomization to evidence of any radiographic disease progression, including local relapse or new pathologic lymph nodes, or death from any cause, whichever occurred first), time to first prostate cancer-related invasive procedure, time to initiation of subsequent antineoplastic therapy, PSA progression and response, deterioration in ECOG performance status, and quality of life. The time to PSA progression from randomization was defined in accordance with Prostate Cancer Working Group 2 (PCWG2) criteria (Table S1 in the Supplementary Appendix). ${ }^{23}$ PSA response was defined as a decline of at least $50 \%$ from baseline in the PSA level, according to PCWG2 criteria. ${ }^{23}$ Deterioration in ECOG performance status was defined as an increase to a score of 3 or higher.

\section{STATISTICAL ANALYSIS}

The sample size was calculated on the basis of the primary end point, metastasis-free survival. Assuming a hazard ratio of 0.71 for death or metastasis in the darolutamide group, we calculated that a sample of 1500 patients (randomly assigned in a 2:1 ratio to receive darolutamide or placebo) with approximately 385 primary end-point events would provide the trial with $91 \%$ power to detect a significant difference in metastasis-free survival with the use of a log-rank test at a two-sided significance level of 0.05 .

The full intention-to-treat population, which was made up of all patients who underwent randomization, was included in the analysis of the primary end point; patients with metastases at baseline were counted as having an event at randomization. Subgroup analyses of metastasis-free survival and overall survival were performed to determine the effect of demographic or baseline characteristics. Randomization stratification factors were used to adjust analyses of the primary and all secondary efficacy end points. Data from patients without events were censored at the last assessment date. Kaplan-Meier curves, including median survival times and their $95 \%$ confidence intervals, were calculated; the hazard ratio was calculated with a Cox proportional-hazards model.

Secondary and exploratory end points were analyzed with the same methods as the primary end point, with the exception of the percentage of patients with PSA response and percentage of patients with deterioration in ECOG performance status, which were analyzed with the CochranMantel-Haenszel test. Secondary end points were evaluated in a hierarchical order, with a significance level of 0.05 split between the primary analysis and final analysis (planned to occur after 240 deaths from any cause) of secondary end points. The end point of overall survival was used to determine the alpha spend and significance threshold for each of the secondary end points. For quality-of-life variables, an analysis of covariance model was used to compare the time-adjusted area under the curve (AUC) between groups, with covariates for baseline scores and randomization stratification factors. The least-squares mean and $95 \%$ confidence interval was estimated for each group and for the difference between the groups.

Statistical analysis and generation of patient data listings were performed with the use of SAS for Windows, version 9.2 (SAS Institute). Incomplete data on event occurrence dates were imputed as the earliest possible date.

\section{RESULTS}

\section{PATIENTS}

Patients were enrolled between September 2014 and March 2018. The intention-to-treat population included 1509 patients (955 in the darolutamide group and 554 in the placebo group); 1 patient in the darolutamide group did not start treatment (Fig. S1 in the Supplementary Appendix). Patient demographic and clinical characteristics were similar in the two trial groups (Table 1). The datacollection cutoff date for the primary analysis was September 3, 2018; the median follow-up time was 17.9 months. At that time, the median duration of the treatment period was 14.8 months in the darolutamide group and 11.0 months in the placebo group, and $64 \%$ of the patients in the darolutamide group and 36\% in the placebo group were still receiving the assigned trial regimen. 


\begin{tabular}{|c|c|c|}
\hline Characteristic & $\begin{array}{l}\text { Darolutamide } \\
\qquad(\mathrm{N}=955)\end{array}$ & $\begin{array}{l}\text { Placebo } \\
(\mathrm{N}=554)\end{array}$ \\
\hline Median age (range) $-y r$ & $74(48-95)$ & $74(50-92)$ \\
\hline \multicolumn{3}{|l|}{ Geographic region — no. (\%) } \\
\hline North America & $108(11)$ & $76(14)$ \\
\hline Asia-Pacific & $119(12)$ & $67(12)$ \\
\hline Rest of the world $\uparrow$ & $728(76)$ & $411(74)$ \\
\hline Median time from initial diagnosis (range) - mo & $86.2(2.6-337.5)$ & $84.2(0.5-344.7)$ \\
\hline \multicolumn{3}{|l|}{ Presence of lymph nodes on central imaging review - no. (\%) } \\
\hline Yes & $163(17)$ & $158(29)$ \\
\hline No & $792(83)$ & $396(71)$ \\
\hline Median serum PSA level (range) — ng/ml & $9.0(0.3-858.3)$ & $9.7(1.5-885.2)$ \\
\hline \multicolumn{3}{|l|}{ PSA doubling time } \\
\hline Median (range) - mo & $4.4(0.7-11.0)$ & $4.7(0.7-13.2)$ \\
\hline$\leq 6 \mathrm{mo}-$ no. (\%) & $667(70)$ & $371(67)$ \\
\hline$>6$ mo - no. (\%) & $288(30)$ & $183(33)$ \\
\hline Median serum testosterone level (range) - nmol/liter & $0.6(0.2-25.9)$ & $0.6(0.2-7.3)$ \\
\hline \multicolumn{3}{|l|}{ ECOG performance status - no. (\%)』 } \\
\hline 0 & $650(68)$ & $391(71)$ \\
\hline 1 & $305(32)$ & $163(29)$ \\
\hline \multicolumn{3}{|l|}{ Use of bone-sparing agent — no. (\%) } \\
\hline Yes & $31(3)$ & $32(6)$ \\
\hline No & $924(97)$ & $522(94)$ \\
\hline \multicolumn{3}{|l|}{ Previous hormonal therapy agents received - no. (\%) } \\
\hline One & $177(19)$ & $103(19)$ \\
\hline Two or more & $727(76)$ & $420(76)$ \\
\hline Not applicable\| & $51(5)$ & $31(6)$ \\
\hline
\end{tabular}

* Percentages may not total 100 because of rounding. PSA denotes prostate-specific antigen.

$\dagger$ This category predominantly includes European countries ( $15 \%$ of these patients came from non-European countries).

$\ddagger$ Testosterone levels from screening or day 1 could be used for eligibility, and all patients met the inclusion criterion of having a testosterone level lower than $1.7 \mathrm{nmol}$ per liter.

$\int$ Eastern Cooperative Oncology Group (ECOG) performance status ranges from 0 to 5, with higher scores reflecting greater disability.

I Common previous hormonal therapies for prostate cancer (received by $\geq 10 \%$ of all patients) included leuprolide (52\%), goserelin (32\%), triptorelin (29\%), bicalutamide (66\%), flutamide (13\%), and cyproterone (11\%).

$\|$ This category includes patients who underwent surgical castration.

\section{PRIMARY END POINT}

The primary analysis of metastasis-free survival was performed after metastasis or death had occurred in 437 patients (Table S2 in the Supplementary Appendix). The median metastasis-free survival was 40.4 months in the darolutamide group, as compared with 18.4 months in the placebo group (hazard ratio for metastasis or death in the darolutamide group, $0.41 ; 95 \%$ confidence interval [CI], 0.34 to $0.50 ; \mathrm{P}<0.001$ ) (Fig. 1A). The treatment effect of darolutamide with regard to metastasis-free survival was consistently favorable across all prespecified subgroups, including in patients with PSA doubling times of 6 months or less or more than 6 months (Fig. 1B).

\section{SECONDARY END POINTS}

Darolutamide was associated with greater benefits than placebo for all secondary end points (Table 2). At this interim analysis of overall sur- 


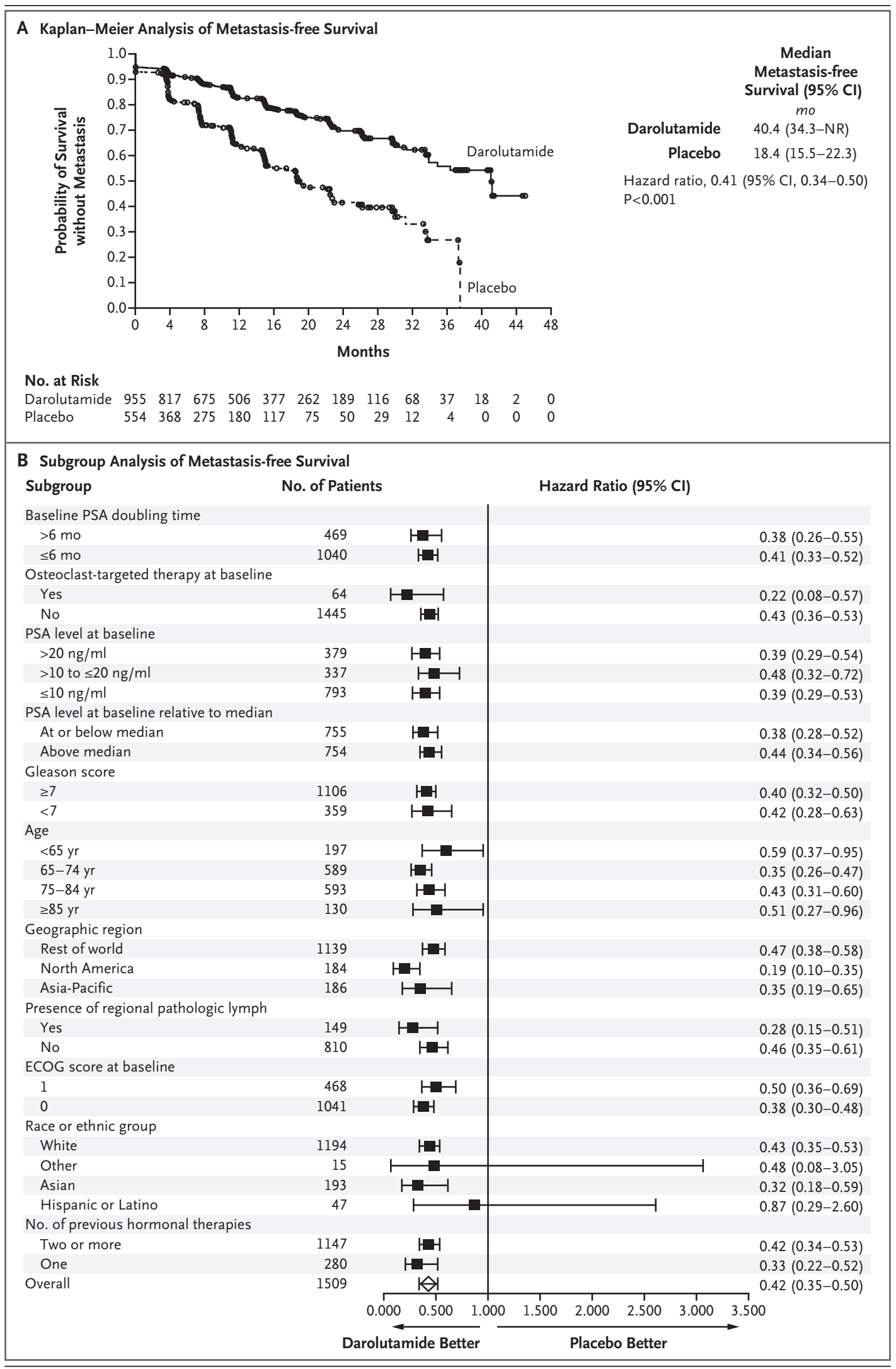


Figure 1 (facing page). Kaplan-Meier Estimates and Subgroup Analyses of Metastasis-free Survival (Intention-To-Treat Population).

Hazard ratios were based on Cox regression models. The analysis shown in Panel A was stratified according to prostate-specific antigen (PSA) doubling time ( $\leq 6$ months or $>6$ months) and the use of osteoclast-targeted therapy at randomization (yes or no). NR denotes not reached. The analyses shown in Panel $B$, including the analysis of the overall population, were conducted without stratification factors. Gleason scores range from 6 to 10, with higher scores indicating higher-risk cancer. The "rest of the world" geographic region was predominantly made up of European countries (15\% of these patients came from non-European countries), and a post hoc analysis of metastasis-free survival in European countries (Austria, Belgium, Germany, Spain, Finland, France, United Kingdom, Italy, Sweden, Portugal, Czech Republic, Estonia, Hungary, Lithuania, Poland, Romania, Russia, Serbia, Turkey, Ukraine, Belarus, Bulgaria, Latvia, and Slovakia) gave a hazard ratio very similar to that for this group. Eastern Cooperative Oncology Group (ECOG) performance status ranges from 0 to 5 , with higher scores reflecting greater disability. The 52 patients of African descent could not be included in the analysis according to race or ethnic group because the number of events was too small to allow calculation of a hazard ratio.

vival after 136 deaths (78 in the darolutamide group and 58 in the placebo group), darolutamide was associated with a lower risk of death than placebo (hazard ratio for death, $0.71 ; 95 \% \mathrm{CI}$, 0.50 to $0.99 ; \mathrm{P}=0.045$ ) (Fig. $2 \mathrm{~A}$ ). The time to pain progression was longer in the darolutamide group than in the placebo group (median, 40.3 months vs. 25.4 months; hazard ratio, 0.65; 95\% CI, 0.53 to $0.79 ; \mathrm{P}<0.001$ ) (Table 2, and Fig. S2A in the Supplementary Appendix). The results with regard to the time to first cytotoxic chemotherapy and time to first symptomatic skeletal event also favored darolutamide (Table 2). Among patients who discontinued the trial regimen, $29.5 \%$ in the darolutamide group and $36.7 \%$ in the placebo group received subsequent approved therapy for metastatic castration-resistant prostate cancer. The most common subsequent treatments were docetaxel, abiraterone acetate, and enzalutamide (Table S3 in the Supplementary Appendix); the frequency of use of abiraterone and enzalutamide was similar across all geographic regions.

\section{EXPLORATORY END POINTS}

Median progression-free survival was 36.8 months in the darolutamide group and 14.8 months in the placebo group (hazard ratio for disease progression or death, 0.38 ; $95 \% \mathrm{CI}, 0.32$ to 0.45 ; $\mathrm{P}<0.001$ ) (Fig. S2B in the Supplementary Appendix). The median time to PSA progression was 33.2 months with darolutamide and 7.3 months with placebo (hazard ratio for PSA progression or death, 0.13 ; $95 \% \mathrm{CI}, 0.11$ to $0.16 ; \mathrm{P}<0.001$ ) (Fig. 2B). The results for other end points also favored darolutamide (Table 2).

Patient-reported quality of life was similar in the darolutamide group and placebo group. Differences in least-squares mean time-adjusted AUC scores consistently favored darolutamide and were significant for BPI-SF (pain severity and pain interference scores), FACT-P (Physical Well-Being, Emotional Well-Being, PCS, General, FACT-P total, and Trial Outcome Index), and the EORTC-QLQPR25 urinary symptoms subscale, although the clinically meaningful thresholds were not reached (Table S4 in the Supplementary Appendix).

\section{SAFETY}

Overall, adverse events were reported by $83.2 \%$ of the patients who received darolutamide and $76.9 \%$ of the patients who received placebo. The majority were grade 1 or $2(54.6 \%$ with darolutamide and $54.2 \%$ with placebo); grade 3 or 4 adverse events occurred in $24.7 \%$ of patients receiving darolutamide and in $19.5 \%$ of those receiving placebo. The incidence of grade 5 adverse events was similar in the darolutamide group and the placebo group (3.9\% and 3.2\%, respectively) (Table S5 in the Supplementary Appendix); one death in the darolutamide group and two deaths in the placebo group were considered to be related to the trial regimen. Serious adverse events occurred in $24.8 \%$ of patients in the darolutamide group and $20.0 \%$ in the placebo group. The percentage of patients who discontinued the assigned regimen because of adverse events was similar in the two groups $(8.9 \%$ in the darolutamide group and $8.7 \%$ in the placebo group) (Table 3 ).

The incidence of adverse events was generally similar in the darolutamide and placebo groups; with the exception of fatigue, all adverse events that occurred or worsened during the treatment period that had a frequency of $5 \%$ or greater occurred in less than $10 \%$ of the patients in either group (Table 3). Key adverse events that are known to be associated with next-generation androgenreceptor inhibitors, such as fracture, falls, seizures, and weight loss, were analyzed after grouping of 


\begin{tabular}{|c|c|c|c|c|c|c|}
\hline \multirow[t]{3}{*}{ End Point } & \multicolumn{2}{|c|}{$\begin{array}{l}\text { Darolutamide } \\
\qquad(\mathrm{N}=955)\end{array}$} & \multicolumn{2}{|c|}{$\begin{array}{l}\text { Placebo } \\
(\mathrm{N}=554)\end{array}$} & \multirow[t]{3}{*}{$\begin{array}{l}\text { Hazard Ratio } \\
(95 \% \mathrm{Cl})\end{array}$} & \multirow[t]{3}{*}{ P Value } \\
\hline & $\begin{array}{l}\text { Median } \\
\text { Duration }\end{array}$ & $\begin{array}{l}\text { No. of } \\
\text { Events }\end{array}$ & $\begin{array}{l}\text { Median } \\
\text { Duration }\end{array}$ & $\begin{array}{l}\text { No. of } \\
\text { Events }\end{array}$ & & \\
\hline & mo & & mo & & & \\
\hline \multicolumn{7}{|l|}{ Secondary end points } \\
\hline Overall survival & NR & 78 & NR & 58 & $0.71(0.50-0.99)$ & 0.045 \\
\hline Time to pain progression & 40.3 & 251 & 25.4 & 178 & $0.65(0.53-0.79)$ & $<0.001$ \\
\hline Time to cytotoxic chemotherapy & NR & 73 & 38.2 & 79 & $0.43(0.31-0.60)$ & $<0.001$ \\
\hline Time to first symptomatic skeletal event & NR & 16 & NR & 18 & $0.43(0.22-0.84)$ & 0.01 \\
\hline \multicolumn{7}{|l|}{ Time-to-event exploratory end points } \\
\hline Progression-free survival & 36.8 & 255 & 14.8 & 258 & $0.38(0.32-0.45)$ & $<0.001$ \\
\hline Time to PSA progression & 33.2 & 226 & 7.3 & 368 & $0.13(0.11-0.16)$ & $<0.001$ \\
\hline $\begin{array}{l}\text { Time to first prostate cancer-related } \\
\text { invasive procedure }\end{array}$ & NR & 34 & NR & 44 & $0.39(0.25-0.61)$ & $<0.001$ \\
\hline $\begin{array}{l}\text { Time to initiation of subsequent anti- } \\
\text { neoplastic therapy }\end{array}$ & NR & 48 & NR & 70 & $0.33(0.23-0.47)$ & $<0.001$ \\
\hline
\end{tabular}

* A total of 798 patients (84\%) in the darolutamide group and $45(8 \%)$ in the placebo group had a PSA response of $50 \%$ or greater. NR denotes not reached.

synonymous or pathophysiologically related adverse events that occurred or worsened during the treatment period; most showed small or no differences in incidence between the darolutamide group and the placebo group. The incidence of seizures was $0.2 \%$ in both groups. Incidences of other adverse events of interest, including hypertension, rash, dizziness, and cognitive disorder, differed only slightly between the darolutamide group and the placebo group. After adjustment for duration of the treatment or observation period, the between-group differences in the incidences of adverse events of interest either decreased or disappeared.

\section{DISCUSSION}

Darolutamide is a nonsteroidal androgen-receptor antagonist that is structurally distinct from other androgen-receptor inhibitors, consisting of two pharmacologically active diastereomers. ${ }^{14}$ In our trial, darolutamide prolonged metastasis-free survival to 40.4 months, 22 months longer than with placebo. The risk of metastasis or death from any cause was reduced by $59 \%$, and the benefit was consistent across all subgroups, including the subgroup of patients with lower-risk disease.
The results for the secondary end point of overall survival also favored darolutamide, although the prespecified alpha split between the primary and the final analysis prevented the significance criteria from being met in this analysis. The results with regard to all secondary end points supported that of the primary end point, and consistent efficacy was observed for metastasis-free, overall, and progression-free survival.

The median metastasis-free survival with darolutamide in the current trial is similar to that in two previous randomized, controlled trials involving patients with nonmetastatic, castrationresistant prostate cancer. The median metastasisfree survival was 36.6 months with enzalutamide (vs. 14.7 months with placebo) in the PROSPER phase 3 trial and was 40.4 months with apalutamide (vs. 16.2 months with placebo) in the SPARTAN (Selective Prostate Androgen Receptor Targeting with ARN-509) phase 3 trial. ${ }^{10,11} \mathrm{Fa}-$ tigue and asthenia, which are common adverse events in patients receiving hormone-targeted therapy for advanced prostate cancer, were less common in the current trial than in the PROSPER or SPARTAN trial. ${ }^{10,11}$ In contrast to apalutamide and enzalutamide, darolutamide was not associated with a higher incidence of falls or fractures 


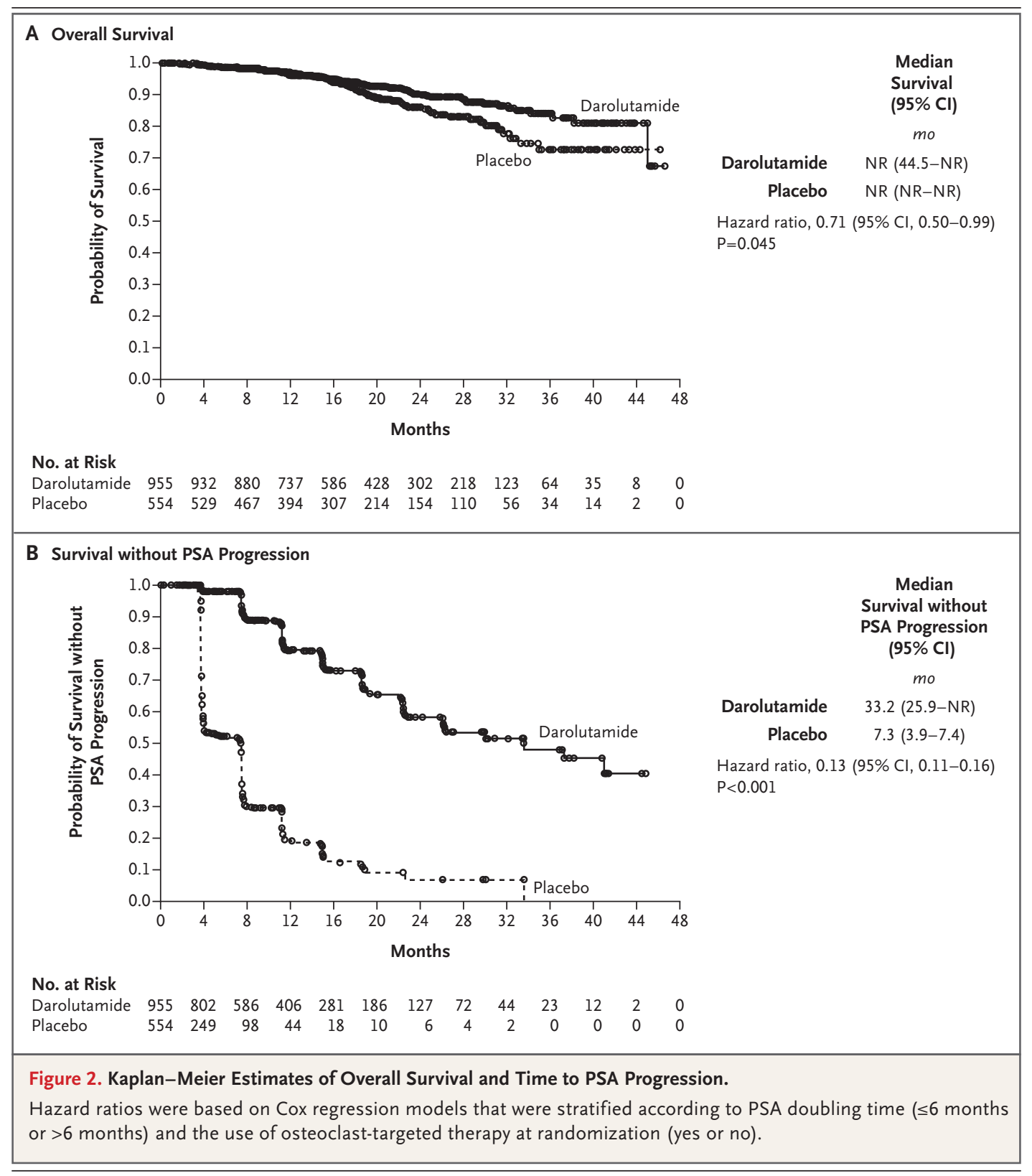

than placebo, ${ }^{10,11}$ despite few patients using osteoclast-targeted therapies. Seizures were noted as a potential risk in the dose-escalation and toxicity studies of enzalutamide, ${ }^{24}$ whereas the preclinical and clinical data for darolutamide did not indicate any proconvulsive potential. ${ }^{19}$ Patients with a history of seizure were therefore allowed to enter the trial, in contrast to the SPARTAN and PROSPER trials. The incidence of seizure events was low and similar in the darolutamide and placebo groups (Table 3); none of the patients with a medical history of seizure (12 in the dar- olutamide group) had a seizure during the trial. The incidences of rash and hypothyroidism, which were higher among patients receiving apalutamide than among those receiving placebo ${ }^{10}$ were low and similar in the darolutamide and placebo groups, as were the incidences of hypertension and central nervous system (CNS)-related adverse events. In the PROSPER and SPARTAN trials, hypertension and CNS-related adverse effects, such as mental-impairment disorders and dizziness, were more common among patients receiving enzalutamide or apalutamide than among those 
receiving placebo. ${ }^{10,11}$ The similar incidences of seizures, dizziness, and cognitive impairment in the darolutamide and placebo groups in the current trial may be linked to the low penetration of the blood-brain barrier that has been found in preclinical studies of the drug. ${ }^{13}$

These results confirm the benefits of early and potent inhibition of androgen-receptor signaling in patients with nonmetastatic, castrationresistant prostate cancer. ${ }^{25}$ Other trials, such as the LATITUDE and STAMPEDE (Systemic Therapy in Advancing or Metastatic Prostate Cancer: Evaluation of Drug Efficacy) trials of abiraterone added to androgen-deprivation therapy in patients with castration-sensitive prostate cancer, have similarly confirmed that early inhibition of androgenreceptor signaling offers significant survival benefits to patients. ${ }^{26,27}$ In addition to survival benefit, quality of life is also an important factor in making treatment choices for patients with nonmetastatic, castration-resistant prostate cancer ${ }^{28}$ since it can be negatively affected by adverse events resulting from therapies and medical interventions as well as from symptomatic disease progression. In the current trial, darolutamide treatment did not adversely affect quality of life, and it resulted in delayed occurrence of metastases with a favorable safety profile.

This trial has several strengths. The large size enabled a robust statistical analysis as well as the detection of rare but important safety signals. Patients' quality of life was assessed in detail with the use of validated instruments to assess different aspects of the effect of treatment, including the BPI scale to measure pain progression. A limitation of the trial is the underrepresentation of patients of African descent (52 in total); therefore, no conclusions can be drawn about efficacy in this group. In addition, treatment with subsequent life-prolonging therapy after the onset of metastases in the placebo group could potentially affect the observed relative benefits of darolu-

\begin{tabular}{|c|c|c|c|c|}
\hline \multirow[t]{3}{*}{ Adverse Event* } & \multicolumn{2}{|c|}{$\begin{array}{l}\text { Darolutamide } \\
\quad(\mathrm{N}=954)\end{array}$} & \multicolumn{2}{|c|}{$\begin{array}{l}\text { Placebo } \\
(\mathrm{N}=554)\end{array}$} \\
\hline & Any Grade & Grade 3 or 4 & Any Grade & Grade 3 or 4 \\
\hline & \multicolumn{4}{|c|}{ number of patients (percent) } \\
\hline Any adverse event & $794(83.2)$ & $236(24.7)$ & $426(76.9)$ & $108(19.5)$ \\
\hline Serious adverse event & $237(24.8)$ & $151(15.8)$ & $111(20.0)$ & $70(12.6)$ \\
\hline Grade 5 adverse event & $37(3.9)$ & - & $18(3.2)$ & - \\
\hline $\begin{array}{l}\text { Adverse event leading to discontinua- } \\
\text { tion of the trial regimen }\end{array}$ & $85(8.9)$ & $32(3.4)$ & $48(8.7)$ & $24(4.3)$ \\
\hline \multicolumn{5}{|l|}{$\begin{array}{l}\text { Adverse events that occurred in } \geq 5 \% \text { of } \\
\text { patients in either group }\end{array}$} \\
\hline Fatigue & $115(12.1)$ & $4(0.4)$ & $48(8.7)$ & $5(0.9)$ \\
\hline Back pain & $84(8.8)$ & $4(0.4)$ & $50(9.0)$ & $1(0.2)$ \\
\hline Arthralgia & $77(8.1)$ & $3(0.3)$ & $51(9.2)$ & $2(0.4)$ \\
\hline Diarrhea & $66(6.9)$ & 0 & $31(5.6)$ & $1(0.2)$ \\
\hline Hypertension & $63(6.6)$ & $30(3.1)$ & $29(5.2)$ & $12(2.2)$ \\
\hline Constipation & $60(6.3)$ & 0 & $34(6.1)$ & 0 \\
\hline Pain in an extremity & $55(5.8)$ & 0 & $18(3.2)$ & $1(0.2)$ \\
\hline Anemia & $53(5.6)$ & $8(0.8)$ & $25(4.5)$ & $2(0.4)$ \\
\hline Hot flush & $50(5.2)$ & 0 & $23(4.2)$ & 0 \\
\hline Nausea & $48(5.0)$ & $2(0.2)$ & $32(5.8)$ & 0 \\
\hline Urinary tract infection & $47(4.9)$ & $6(0.6)$ & $28(5.1)$ & $3(0.5)$ \\
\hline Urinary retention & $33(3.5)$ & $15(1.6)$ & $36(6.5)$ & $11(2.0)$ \\
\hline
\end{tabular}




\begin{tabular}{|c|c|c|c|c|}
\hline \multirow[t]{2}{*}{ Adverse Event** } & \multicolumn{2}{|c|}{$\begin{array}{l}\text { Darolutamide } \\
\qquad(\mathrm{N}=954)\end{array}$} & \multicolumn{2}{|c|}{$\begin{array}{l}\text { Placebo } \\
(\mathrm{N}=554)\end{array}$} \\
\hline & \multicolumn{4}{|c|}{ number of patients (percent) } \\
\hline Adverse events of interest & & & & \\
\hline Fatigue or asthenic conditions $\uparrow$ & $151(15.8)$ & $6(0.6)$ & $63(11.4)$ & $6(1.1)$ \\
\hline Bone fracture & $40(4.2)$ & $9(0.9)$ & $20(3.6)$ & $5(0.9)$ \\
\hline Falls, including accident $\llbracket$ & $40(4.2)$ & $8(0.8)$ & $26(4.7)$ & $4(0.7)$ \\
\hline Seizure, any event & $2(0.2)$ & 0 & $1(0.2)$ & 0 \\
\hline Rash & $28(2.9)$ & $1(0.1)$ & $5(0.9)$ & 0 \\
\hline Weight decrease, any event & $34(3.6)$ & 0 & $12(2.2)$ & 0 \\
\hline Dizziness, including vertigo & $43(4.5)$ & $2(0.2)$ & $22(4.0)$ & $1(0.2)$ \\
\hline Cognitive disorder & $4(0.4)$ & 0 & $1(0.2)$ & 0 \\
\hline Memory impairment & $5(0.5)$ & 0 & $7(1.3)$ & 0 \\
\hline Change in mental status & 0 & 0 & $1(0.2)$ & 0 \\
\hline Hypothyroidism & $2(0.2)$ & 0 & 0 & 0 \\
\hline Cerebral ischemia $\|$ & $13(1.4)$ & $7(0.7)$ & $8(1.4)$ & $4(0.7)$ \\
\hline Coronary-artery disorder** & $31(3.2)$ & $16(1.7)$ & $14(2.5)$ & $2(0.4)$ \\
\hline Heart failureti & $18(1.9)$ & $5(0.5)$ & $5(0.9)$ & 0 \\
\hline
\end{tabular}

* Exposure-adjusted incidences of adverse events in the darolutamide group and the placebo group were as follows: fatigue or asthenic conditions (11.3 patients per 100 years of exposure and 11.1 patients per 100 years of exposure, respectively), back pain (6.3 and 8.8), arthralgia (5.8 and 9.0), diarrhea (4.9 and 5.5), hypertension (4.7 and 5.1), constipation (4.5 and 6.0), pain in extremity (4.1 and 3.2), anemia (4.0 and 4.4), hot flush (3.7 and 4.1), nausea (3.6 and 5.6), weight loss (2.5 and 2.1), falls (2.7 and 4.1), bone fracture (3.0 and 3.5), memory impairment (0.4 and 1.2$)$, cognitive disorder (0.3 and 0.2$)$, and seizure $(0.2$ and 0.2 ).

$\dagger$ This category combines the following Medical Dictionary for Regulatory Activities, version 20.0 (MedDRA) terms: asthenic conditions, disturbances in consciousness, decreased strength and energy, malaise, lethargy, asthenia, and fatigue.

$\dagger$ This category combines the following MedDRA terms: any fractures and dislocations, limb fractures and dislocations, skull fractures, facial bone fractures and dislocations, spinal fractures and dislocations, and thoracic cage fractures and dislocations.

$\int$ All events that had been recorded under the MedDRA term "accident" were determined to have been accidental falls and are included in this category.

I This category combines the following MedDRA terms: dermatitis, erythema, rash, macular rash, maculopapular rash, papular rash, and pustular rash.

\| This category combines the following MedDRA terms: cerebral infarction, cerebral ischemia, cerebrovascular accident, ischemic stroke, and transient ischemic attack. Grade 5 events occurred in one patient receiving darolutamide and three patients receiving placebo.

** This MedDRA High Level Group Term includes coronary-artery disorders not elsewhere classified, coronary-artery arteriosclerosis, coronary artery disease, coronary-artery occlusion, and coronary-artery stenosis. Grade 5 events occurred in three patients receiving darolutamide and one patient receiving placebo.

t† This MedDRA High Level Group Term includes heart failure not elsewhere classified, cardiac failure, acute cardiac failure, chronic cardiac failure, congestive cardiac failure, and cardiogenic shock. Grade 5 events occurred in four patients receiving darolutamide and three patients receiving placebo.

tamide with regard to secondary end points. The percentages of patients who received subsequent treatment are lower than those reported in the SPARTAN trial, in which the trial design included the provision of abiraterone for patients in whom metastases developed.
In conclusion, metastasis-free survival was significantly longer with darolutamide than with placebo for men with nonmetastatic, castrationresistant prostate cancer and a PSA doubling time of 10 months or less. The results for the secondary and exploratory end points supported the 
benefits of darolutamide in this clinical context. The safety data indicated no clinically relevant difference between darolutamide and placebo in the incidence of adverse events that occurred during the treatment period, including falls, fractures, seizures, cognitive disorders, and hypertension. Quality-of-life outcomes were similar in the two groups.
Supported by Bayer HealthCare and Orion Pharma.

Disclosure forms provided by the authors are available with the full text of this article at NEJM.org.

A data sharing statement provided by the authors is available with the full text of this article at NEJM.org.

We thank the patients and their families, all the investigators involved in this trial, and Lucy Smithers, Ph.D., and Annabel Ola, M.Sc., both of Scion, London, for medical writing and editing support (supported by Bayer).

\section{REFERENCES}

1. NCCN clinical practice guidelines in oncology: prostate cancer, version 4. Plymouth Meeting, PA: National Comprehensive Cancer Network, 2018 (https:// www.nccn.org/professionals/physician _gls/default.aspx\#site).

2. Gillessen S, Attard G, Beer TM, et al. Management of patients with advanced prostate cancer: the report of the advanced prostate cancer consensus conference APCCC 2017. Eur Urol 2018;73:178-211.

3. Bolla M, Gonzalez D, Warde P, et al. Improved survival in patients with locally advanced prostate cancer treated with radiotherapy and goserelin. N Engl J Med 1997;337:295-300.

4. Crook JM, O'Callaghan CJ, Duncan G, et al. Intermittent androgen suppression for rising PSA level after radiotherapy. N Engl J Med 2012;367:895-903.

5. Anantharaman A, Small EJ. Tackling non-metastatic castration-resistant prostate cancer: special considerations in treatment. Expert Rev Anticancer Ther 2017;17: 625-33.

6. Smith MR, Kabbinavar F, Saad F, et al. Natural history of rising serum prostatespecific antigen in men with castrate nonmetastatic prostate cancer. J Clin Oncol 2005;23:2918-25.

7. Halabi S, Kelly WK, Ma H, et al. Metaanalysis evaluating the impact of site of metastasis on overall survival in men with castration-resistant prostate cancer. J Clin Oncol 2016;34:1652-9.

8. Gravis G, Boher JM, Joly F, et al. Androgen deprivation therapy (ADT) plus docetaxel versus ADT alone in metastatic non castrate prostate cancer: impact of metastatic burden and long-term survival analysis of the randomized phase 3 GETUG-AFU15 trial. Eur Urol 2016;70: 256-62.

9. Xie W, Regan MM, Buyse $M$, et al. Metastasis-free survival is a strong surrogate of overall survival in localized prostate cancer. J Clin Oncol 2017;35:3097-104. 10. Smith MR, Saad F, Chowdhury S, et al. Apalutamide treatment and metastasisfree survival in prostate cancer. $\mathrm{N}$ Engl J Med 2018;378:1408-18.

11. Hussain M, Fizazi K, Saad F, et al. Enzalutamide in men with nonmetastat- ic, castration-resistant prostate cancer. N Engl J Med 2018;378:2465-74.

12. Sandmann S, Trummel D, Seidel D Nubbemeyer R, Gieschen H, Zurth C. Higher blood-brain barrier penetration of [14C]apalutamide and [14C]enzalutamide compared to [14C]darolutamide in rats using whole-body autoradiography. Presented at the ASCO Genitourinary Cancers Symposium, San Francisco, February 14-16, 2019. abstract.

13. Zurth C, Sandmann S, Trummel D, Seidel D, Gieschen H. Blood-brain barrier penetration of [14C]darolutamide compared with [14C]enzalutamide in rats using whole body autoradiography. J Clin Oncol 2018;36:Suppl 6S:345. abstract.

14. Moilanen AM, Riikonen R, Oksala R, et al. Discovery of ODM-201, a new-generation androgen receptor inhibitor targeting resistance mechanisms to androgen signaling-directed prostate cancer therapies. Sci Rep 2015;5:12007.

15. Moilanen AM, Riikonen R, Oksala R, et al. ODM-201 - new generation androgen receptor inhibitor with excellent antiandrogenic and antitumor activity in nonclinical models of CRPC. Eur J Cancer 2013;49:Suppl 2:S685. abstract.

16. Fizazi K, Massard C, Bono P, et al. Activity and safety of ODM-201 in patients with progressive metastatic castrationresistant prostate cancer (ARADES): an open-label phase 1 dose-escalation and randomised phase 2 dose expansion trial. Lancet Oncol 2014;15:975-85.

17. Fizazi K, Massard C, Bono P, et al. Safety and antitumour activity of ODM201 (BAY-1841788) in castration-resistant, CYP17 inhibitor-naïve prostate cancer: results from extended follow-up of the ARADES trial. Eur Urol Focus 2017;3: 606-14.

18. Massard C, Penttinen HM, Vjaters E, et al. Pharmacokinetics, antitumor activity, and safety of ODM-201 in patients with chemotherapy-naive metastatic castrationresistant prostate cancer: an open-label phase 1 study. Eur Urol 2016;69:834-40.

19. Shore ND, Tammela TL, Massard C, et al. Safety and antitumour activity of ODM-201 (BAY-1841788) in chemotherapy-naïve and CYP17 inhibitor-naïve pa- tients: follow-up from the ARADES and ARAFOR trials. Eur Urol Focus 2018;4: 547-53.

20. Cella D, Nichol MB, Eton D, Nelson JB, Mulani P. Estimating clinically meaningful changes for the Functional Assessment of Cancer Therapy - Prostate: results from a clinical trial of patients with metastatic hormone-refractory prostate cancer. Value Health 2009;12:124-9.

21. van Andel G, Bottomley A, Fosså SD, et al. An international field study of the EORTC QLQ-PR25: a questionnaire for assessing the health-related quality of life of patients with prostate cancer. Eur J Cancer 2008;44:2418-24.

22. Common Terminology Criteria for Adverse Events (CTCAE), version 4.03. Bethesda, MD: Cancer Therapy Evaluation Program, 2010 (https://ctep.cancer.gov/ protocoldevelopment/electronic _applications/ctc.htm).

23. Scher HI, Halabi S, Tannock I, et al. Design and end points of clinical trials for patients with progressive prostate cancer and castrate levels of testosterone: recommendations of the Prostate Cancer Clinical Trials Working Group. J Clin Oncol 2008;26:1148-59.

24. Scher HI, Beer TM, Higano CS, et al. Antitumour activity of MDV3100 in castration-resistant prostate cancer: a phase 1-2 study. Lancet 2010;375:1437-46.

25. Mateo J, Fizazi K, Gillessen S, et al. Managing nonmetastatic castration-resistant prostate cancer. Eur Urol 2019;75: 285-93.

26. Fizazi K, Tran N, Fein L, et al. Abiraterone plus prednisone in metastatic, castration-sensitive prostate cancer. N Engl J Med 2017;377:352-60.

27. James ND, de Bono JS, Spears MR, et al. Abiraterone for prostate cancer not previously treated with hormone therapy. N Engl J Med 2017;377:338-51.

28. Foucher Y, Lorent M, Tessier P, Supiot S, Sébille V, Dantan E. A mini-review of quality of life as an outcome in prostate cancer trials: patient-centered approaches are needed to propose appropriate treatments on behalf of patients. Health Qual Life Outcomes 2018;16:40.

Copyright (C) 2019 Massachusetts Medical Society. 